\title{
General framework for techno-economic analysis of next generation access networks
}

\author{
Miroslaw Kantor, Krzysztof Wajda, Bart Lannoo*, Koen Casier*, Sofie Verbrugge*, Mario Pickavet*, \\ Lena Wosinska**, Jiajia Chen**, Attila Mitcsenkov*** \\ Dep. of Telecomm., AGH University of Science and Technology, Al. Mickiewicza 30, 30-059 Krakow, Poland \\ *Dep. of Information Technology, Ghent University - IBBT, Gaston Crommenlaan 8, B-9050 Gent, Belgium \\ ** Royal Institute of Technology (KTH), School of ICT, S-164 40 Kista, Sweden \\ ***Dept. of Telecomm. and Media Informatics, Budapest Uni. of Technology and Economics, Hungary \\ (Invited Paper) \\ Tel: (48) 12 6172852,Fax: (48)126342372,e-mail: kantor@kt.agh.edu.pl
}

\begin{abstract}
A large variety of access network technologies and architectures that provide wide service portfolio to the customer are available for the network operators. Each of the potential access network architectures and technologies varies in complexity, network functionality, services supported and overall network costs. A detailed comparison of the economic viability of different access network scenarios is crucial for operators due to the high cost of this network segment. This paper identifies all essential elements of a general framework for the techno-economic analysis of different access network technologies and architectures, as well as describes some specific issues/problems related to the techno-economic evaluation of next generation (NG) access networks. The goal is to have at operator's disposal a methodology allowing the techno-economic comparison of the proposed access network solutions and their introduction/rollout.
\end{abstract}

Keywords: methodology, access network, techno-economic analysis

\section{INTRODUCTION}

In order to handle the emerging demands for broadband services, adequate telecommunication access network designs are crucial for network operators, service providers and equipment vendors. As a number of technical candidates and design options for constructing access networks exist, it is necessary to perform calculations to identify cost-efficient combinations of technologies, functionalities and network structures. For this reason, a large variety of access network architectures have to be checked in order to determine the most appropriate ones for different area types and service demand profiles. Moreover, besides technical options, other issues like regulatory and competitive aspects should be taken into account. An accurate construction of a techno-economic model allows to minimize errors in the network development phase and to calculate intermediate results, allowing an evolutionary development of the network solution. The detailed modelling, including offered services, serving area, equipment, operational cost processes, revenues and other related techno-economic elements, assures a significant conformity between techno-economic models and real deployment.

New infrastructures and equipment are often required in order to deliver broadband services to the end users. Optical fibre networks are considered as the most future-proof next generation (NG) access technologies. Nowadays, the most used optical fibre access network configuration is a (power splitting) time division multiplexing (TDM) passive optical network (PON), with gigabit-capable PON (GPON) and Ethernet PON (EPON) as the two most important standards [1-2]. Wavelength division multiplexing (WDM) PONs and hybrid WDM/TDM PONs can be considered as strong candidates for next-generation PONs (NG-PONs) [3]. Next to PON architectures, also active optical networks (AON) offer a possible NG access solution.

In literature some techno-economic assessment methodologies have been proposed [4-6]. Our framework is the extension of the existing methodologies. Taking into account technical parameters in the techno-economic framework helps to deploy cost-efficient access network architectures with required technical performance. The objective of the paper is to identify all the essential elements of such techno-economic model

\section{FRAMEWORK AND METHODOLOGY}

Figure 1 gives an overview of the methodology for performing a complete techno-economic evaluation. It starts from determining the scope of the problem and detailing the inputs for the study based on a market analysis. The most important outcomes here are indicated by the building blocks services and architectures. They contain all input information necessary for building the techno-economic model in the second step. Often in a telecom project the network is the central piece and contains most optimization opportunities. Moreover, a proper network design also reflects suitability of a certain network infrastructure to the considered scenario, and therefore it supports the optimal choice among the competing technologies. As such, network design is given a central position in this figure as the link between market analysis and calculations. In the calculations we make a distinction between economic calculations, in which we estimate costs and revenues; and the technical calculations, in which we estimate the performance metrics of the proposed network solution. In the final step, 
an evaluation will be based on the outcomes - economic and technical - of the calculations step. This step is split between investment analysis and performance analysis. In the first part, we make an estimation of the (expected) profitability of the project. In the second part, we compare different alternatives and make tradeoffs of costs vs. performance. Both results are the final outcome of a well balanced techno-economic study.

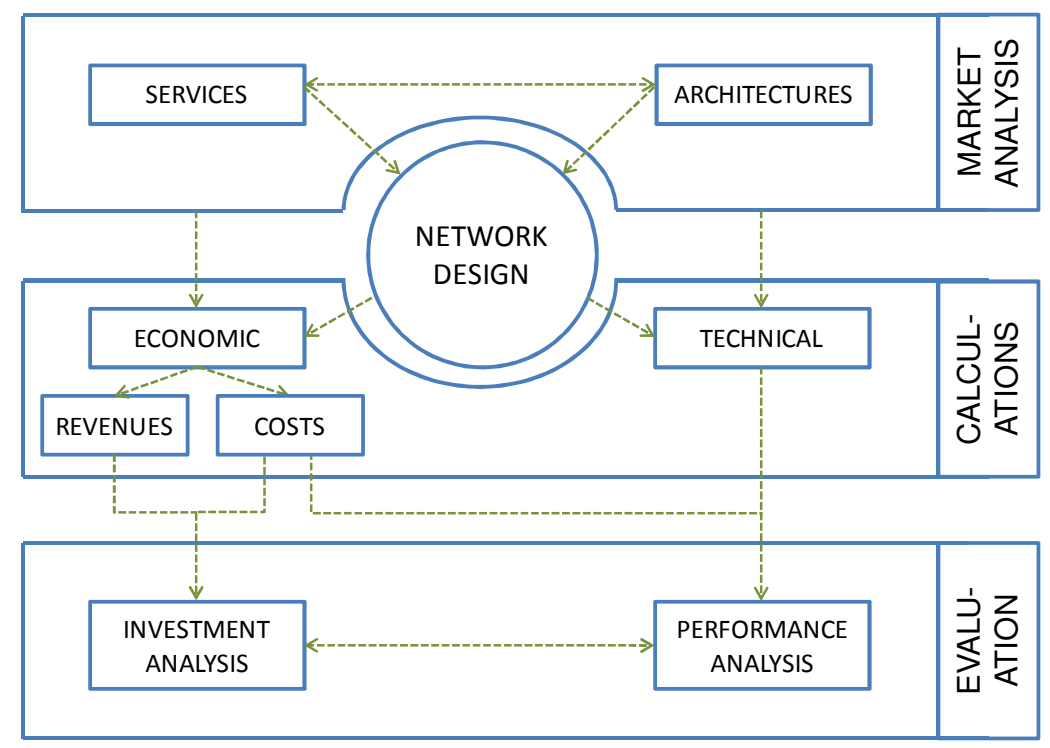

Figure 1: Flow diagram of a techno-economic evaluation

\section{MARKET ANALYSIS FOR THE TECHNO-ECONOMIC FRAMEWORK}

Market analysis related to tariffs, regulations and market shares is a first step in the techno-economic framework. Different tariffs and fees can be assigned to different applications and customer groups. A proper market model should also contain information on the regulatory context, competitive issues, customer preferences, etc.

\subsection{Services}

Demand modelling and broadband forecasts for considered access technologies are essential inputs to all business case analyses. These aspects take into account issues related to customers, applications demands and their characteristics. A number of potential customers has to be described, together with a set of available applications. Each application has to be described by a set of parameters, e.g., bandwidth, usage intensity, quality and resilience requirements leading to engineering rules, and e.g., cost, subscription options and type of service agreement leading to pricing consequences. The service scenario should be prepared defining how many subscribers are connected to a certain service year by year and how much revenue brings one service user for the network operator per year. The penetration of the service, meant as a number of users in time, and the usage of the service also have to be taken into account in techno-economic analysis. These parameters can be estimated from historical data and from forecasts.

\subsection{Architectures}

The description of relevant technologies, systems and architectures to provide the services in the form of selected target access network architectures should be given. In case the access network migration path is considered, also the details related to intermediate scenarios have to be defined. Based on the assumed access network evolution path, the amount of equipment needed in the network and the time when the various components actually are implemented should be indicated.

The cost figures for the network components should be based on comparable input data (with respect to volumes, market, etc.) and collected in an integrated cost database. The cost evolution for the actual components during the study period should also be taken into account, e.g., by applying a learning curve model [7]. Apart from the cost figures, also the technical parameters, such as bandwidth capacity, power consumption, reach, mean time between failures (MTBF), mean time to repair (MTTR), etc. should be provided.

The final step based on the architectures and services should be describing the deployment rules aiming at economically and technically justified network dimensioning and management.

\section{NETWORK DESIGN}

Network design plays a central role in techno-economic calculations. From the network design, the lengths of fibres, cables and ducts are determined. Additionally, in view of the planned network topology, detailed lists of 
the necessary network equipment and installation works are available that support accurate, realistic preliminary cost estimations; and also OpEx calculation models are heavily dependent on the network topology itself. On the other hand, designing the access network is impossible without the market analysis described in the previous section, as the chosen network architecture obviously sets constraints on the network topology that has to serve all the customer demand and provide the desired services.

Impact of the physical network infrastructure is decisive: no "overall best" network architecture exists. Finding the most suitable solution for a given service area, service set and economic conditions plays a crucial role regarding profitability of the network operator - and all these aspects strongly determine or are determined by the network design. The physical access network could be represented by analytical models [8] using overall parameters of the selected area (e.g. population density, diameter); or the results of a realistic, geography-based network design process could be used directly, supposed that the required geospatial information is available [9].

\section{CALCULATION DETAILS}

In order to make a techno-economic analysis of the full project, different techno-economic calculations must be performed. At first an economic model will give an estimation of all costs and revenues the project will generate. Additional technical calculations will give more information on the performance of the outcome of the project under consideration. Both parts are discussed in more detail in the following subsections.

\subsection{Economic calculation}

In order to cover all costs while building the economic model, it is good practice to start from a life-cycle of the project and perform a gradual cost-breakdown for the costs of the different phases. Figure 2 shows the life-cycle cost for a typical FTTH network rollout [10].

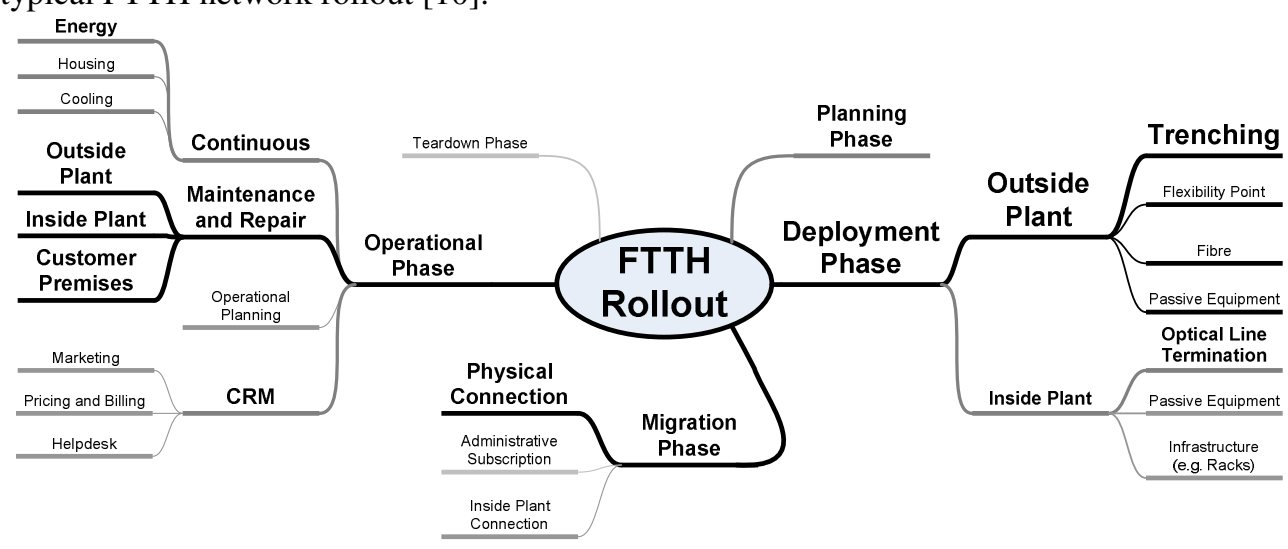

Figure 2: Capital and operational costs for the rollout cycle of an FTTH network [10]

This lifecycle additionally indicates which level of detail to use for the estimation of each part. By providing an (estimative) calculation model for each of the components in this lifecycle a full economic model is built. Finally it is important to note that we make a distinction between infrastructure and operational cost components, based on their origin. This distinction makes sense as both have typically a different structure, information sources and calculation approach. Infrastructure costs are modelled using equipment models and will take specifics of this equipment, such as failure-rates and replacement periods into account. Infrastructure is also typically bundled under Capital Expenditures (CapEx) and can be depreciated. Operational costs are modelled using manpower and operational processes and are typically bundled under Operational Expenditures (OpEx).

In combination with the revenues the economic model is complete. Revenues are typically easier to calculate, based on the subscription rate and a description of the market (market size, service penetrations). The market size in turn is estimated using a so-called customer adoption model [11] or dedicated market research figures.

\subsection{Technical calculations}

Based on the input from the market analysis and network design, performance parameters such as capacity, energy consumption, reach, reliability and other technical indicators can be calculated. These technical parameters are then essential for techno-economic evaluation.

One of the most important objectives of NG access networks is to satisfy an increasing bandwidth demand from the end users. Large capacity (more than $1 \mathrm{Gbp} / \mathrm{s}$ per customer) is a crucial technical criterion for NG access network solutions. Energy consumption by the ICT sector is rapidly growing and becomes an environmental, social and political issue. Therefore, power consumption per bit and per customer in access networks should be minimized in order to contribute to the overall energy saving in communication networks. Extending the reach of access networks from a few kilometres to about 100 kilometres allows for a site reduction and, consequently, gives a potential for lowering the OpEx. However, some technical issues are associated with the reach extension 
since the power budget needs to be satisfied. Therefore, the maximum distance supported by a given access network architecture is an important parameter. The importance of fault management and reliability performance of access networks increases with the network size and capacity as well as with the new and emerging services. Therefore, network monitoring and recovery mechanisms are essential for the reliable service delivery. Consequently, the reliability performance of candidate access network architectures needs to be evaluated.

\section{TECHNO-ECONOMIC EVALUATION}

The techno-economic evaluation incorporates results from both investment and performance analysis with the aim of selecting the most cost-efficient solution for a certain scenario and performance requirements.

\subsection{Investment analysis}

Typical investment analysis will combine all cash flows (costs and revenues in time) and make a decision on the profitability of the investment project. Key performance indicators are Cash balance, Net Present Value (NPV), Internal Rate of Return (IRR) for a given interest rate. As the NPV gives the best view on the economic value of the whole project we typically use this as the deciding metric and use the others as indicative additions.

\subsection{Performance analysis}

Based on the economical and technical calculations, techno-economic performance indicators can be evaluated. One of the performance parameters is the cost per capacity unit for each user. It can be a more indicative measure than the cost per user as it would allow for a fair comparison of different access technologies. The relation between cost and reliability also should be evaluated. A large variety of reliable network architectures has been developed in the recent years with the aim to offer high service availability in NG access networks [12]. On the other hand, improvement of reliability performance is typically associated with additional investment cost while access network providers need to keep CapEx and OpEx at reasonably low level to be able to offer economical solutions. Therefore, a techno-economic analysis which takes into account the resilience issues helps to select the most cost-efficient approach in respect to the reliability performance of an access network.

\section{CONCLUSIONS}

In this paper we have taken into account the technical and business aspects of building broadband access networks. All essential cost elements, including both capital and operational expenditures have been indicated and described. Any business modelling should be accompanied by techno-economic evaluation in order to give readers insights into the financial perspective and technical viability of a telecommunication investment project.

\section{ACKNOWLEDGEMENTS}

The work described in this paper was carried out with the support of the BONE-project ("Building the Future Optical Network in Europe"), a Network of Excellence funded by the European Commission through the 7th ICT-Framework Programme.

\section{REFERENCES}

[1] IEEE 802.3ah task force home page [Online]. Available: http://www.ieee802.org/3/efm.

[2] ITU-T G.984.x series of recommendations [Online]. Available: http://www.itu.int/rec/T-REC-G/e.

[3] K. Grobe and J. P. Elbers, "PON in Adolescence: From TDMA to WDM-PON", IEEE Communications Magazine, vol. 46, pp. 26-34, Jan. 2008.

[4] B. Olsen et al., "Techno-economic Evaluation of the Major Telecommunication Investment Options for European Players", IEEE Network, pp. 6-15, July/August 2006.

[5] S. Verbrugge et al., "Methodology and input availability parameters for calculating OpEx and CapEx costs for realistic network scenarios", Journal of Optical Networking, Vol. 5, Issue 6, pp. 509-520, 2006.

[6] T. Monath et al., "Techno-Economic aspects of Fixed-Mobile Convergence (FMC) opportunities based on the MUSE project", in Proc. Broadband Europe 2007, Antwerp, Belgium, December 3-6, 2007.

[7] B. Olsen et al., Telektronikk, 100 (4), 138-148, 2004.

[8] D. Gardan et al., "Techno-economics of advanced optical subscriber networks", in Proc. IEEE GLOBECOM'89, 1989.

[9] A. Mitcsenkov et al., "Topology Design and Capex Estimation for Passive Optical Networks", in Proc. BroadNets2009, Madrid, Spain, September 14-16, 2009.

[10] K. Casier et al., "Techno-economic evaluations of FTTH roll-out scenarios", in Proc. NOC2008, pp. 113120, Krems, Austria, July 1-3, 2008.

[11] K. Casier et al., "Adoption and Pricing; the Underestimated Elements of a Realistic IPTV Business Case", IEEE Communications Magazine, Vol. 46, pp. 112-118, August 2008.

[12] J. Chen and L. Wosinska, "Analysis of protection schemes in PON compatible with smooth migration from TDM-PON to hybrid WDM/ TDM-PON”, OSA Journal of Optical Networking, Vol. 6, No 5, 2007. 